\title{
Fractal Analysis on Asphalt Mixture Using a Two-Dimensional Imaging Technique
}

\author{
Yue Hou, ${ }^{1}$ Yucheng Huang, ${ }^{2}$ Fengyan Sun, ${ }^{1}$ and Meng Guo ${ }^{1}$ \\ ${ }^{1}$ National Center for Materials Service Safety, University of Science and Technology Beijing, Beijing, China \\ ${ }^{2}$ The Via Department of Civil and Environmental Engineering, Virginia Polytechnic Institute and State University, Blacksburg, \\ VA 24061, USA \\ Correspondence should be addressed to Fengyan Sun; fysun@ustb.edu.cn
}

Received 3 August 2016; Accepted 10 October 2016

Academic Editor: Katsuyuki Kida

Copyright (C) 2016 Yue Hou et al. This is an open access article distributed under the Creative Commons Attribution License, which permits unrestricted use, distribution, and reproduction in any medium, provided the original work is properly cited.

\begin{abstract}
Fractal is a mathematical set that has a fractal dimension which usually exceeds its topological dimension and may be nonintegral. Since the asphalt pavement texture has limitations of randomness and self-similarity, fractal theory has been explored to quantify the asphalt pavement texture and employs good applicability in processing and analyzing the complex details of research object. In this paper, the 2D digital image of the pavement surface is measured in terms of area fractal dimension and contour fractal dimension, which are used to correlate with aggregate gradation and British Pendulum Number (BPN) value, respectively. It turns out the area fractal dimension of aggregate provides a simple way to acquire the continuous gradation of asphalt concrete sample and the contour fractal dimension is an available parameter to characterize roughness and friction of pavement surface texture.
\end{abstract}

\section{Introduction}

Fractal is a mathematical set that has a fractal dimension which usually exceeds its topological dimension and may be nonintegral $[1,2]$. Fractals are typically self-similar patterns, where self-similarity means they are "the same from near as from far" [3]. The American mathematician Mandelbort first proposed the fractal dimension and fractal geometry theory in 1973, offering a quite new idea for fitting data, which can characterize a large number of rough curves and curved surfaces existing in nature in a better way [3]. The fractal interpolation has been a prevalent application in various data visualization coupled with model reconstruction and simulation; for example, Mandelbort used an iterated function system (IFS) generated by a set of affine transformations to build fractal interpolation function (FIF) interpolating a set of nodes on the planes [4]. The initial study object of fractal geometry is a subset of the class of complex structures within Euclidean geometry. However, with the development of fractal geometry, as a complicated phenomenon in the natural science research, the fractal interpolation method has been widely studied.
In terms of sizes and shapes of aggregates and surface texture of asphalt pavement, the mechanical and engineering properties are characterized by uncertainty, irregularity, fuzziness, and nonlinearity; however, the obvious randomness and complexity with self-similarity of the asphalt pavement texture, fractal theory has been explored to quantify the asphalt pavement texture and employs good applicability in processing and analyzing the complex details of research object. Arasan et al. [5] conducted a study of the influence of fractal dimension on mechanical properties of asphalt concrete and found that Marshall Stability is proportional to the fractal dimension of aggregate increases. Villani et al. [6] evaluated pavement surface friction property by the means of fractal analysis. Dong et al. [7] and Liu and Wang [8] applied fractal analysis to study the gradation of asphalt concrete, concluded the relationship between fractal dimension and performance of asphalt mixture, and recommended the fractal dimension value of asphalt concrete (AC) gradation with good performance. Although there have been some progress in the asphalt pavement and mixture area [911], in the previous studies, most researches are focused on the volumetric fractal dimension of the asphalt mixture 
gradation and it is very hard to obtain the gradation data of a formed asphalt concrete. In this paper, a simple means is proposed to approximately estimate the gradation of the asphalt mixture through the digital images. Note that our current research scale is between microscale and macroscale, which needs to be further refined and developed in future studies considering the current multiscale research progress in asphalt pavement areas $[12,13]$.

\section{Fractal Dimension Calculation}

Fractal dimension measurements are used to estimate and quantify the complexity of form, shape, or texture of objects [14]. Although many sorts of algorithms have been developed for computing fractal dimension based on digital image processing, this paper employs the box-counting method. The box-counting method is one of the most popular methods to compute fractal dimension because of its simplicity in operation and understanding. The box-counting method is based on the number of boxes $N(\varepsilon)$ with the size $\varepsilon$ required to fill the entire area of the object. With the change of the size $\varepsilon$, the number of boxes $N(\varepsilon)$ also changes. In other words, the smaller the size of $\varepsilon$, the larger the number of boxes, while the larger the size of $\varepsilon$, the fewer the number of boxes $N(\varepsilon)$. The box-counting method defines the fractal dimension of an object by the expression in (1) as follows [15]:

$$
D=\lim _{\varepsilon \rightarrow 0}\left\{\frac{\log (N(\varepsilon))}{\log (1 / \varepsilon)}\right\} .
$$

\section{Fractal Dimension of Pavement Surface Profile}

In macroscale of pavement engineering, the parameter IRI (International Roughness Index) is used to represent the roughness of the pavement; however, there is no parameter to characterize the roughness of sample surface in microscale. Therefore, fractal dimension is considered to be a relevant parameter to evaluate the roughness of surface profile. To evaluate the asphalt pavement macrotexture characteristics, 37 test sites located in Chaoyang and Huairou Districts in Beijing, China, are selected for collecting 3D macrotexture data. Figure 1 presents the surface textures of five different types of pavements: asphalt concrete (AC), microsurfacing (MS), Open Grade Friction Course (OGFC), Rubber AC (RAC), and Stone Mastic Asphalt (SMA). The length of each sample is $20 \mathrm{~cm}$. The height of each data point was captured at the interval of $0.5 \mathrm{~mm}$ by a laser scanner.

Table 1 presents the fractal dimension calculated with MATLAB program and the variance of the data. The variance was calculated to represent the roughness of the surface. Figure 2 indicates the relationship between fractal dimension and variance of the five different types of pavement samples. Based on Figure 2, the fractal dimension increases with the growth of variance. Hence, the fractal dimension can characterize the roughness of the profiles.

The procedures of fractal analysis by MATLAB program code are shown in Figure 3.

Figure 3 shows the process of image analysis with the MATLAB program. First, the original image should be
TABLE 1: Fractal dimension and variance of 5 samples.

\begin{tabular}{lccccc}
\hline & MS & AC & SMA & RAC & OGFC \\
\hline Variance & 0.0616 & 0.287 & 0.3052 & 0.659 & 1.2138 \\
FD & 1.0058 & 1.0185 & 1.025 & 1.043 & 1.102 \\
\hline
\end{tabular}

converted to the gray scale. Second, due to the little difference between the aggregates and background, the contrast of the picture needs to be modified to find the appropriate threshold value. There are some filter functions applied in the program to delete the noises in the picture. Third, the gray scale image is converted to binary image to calculate area fractal dimension $\left(D_{a}\right)$. The contour fractal dimension $\left(D_{c}\right)$ can be obtained after exploiting functions to depict the boundary of the aggregates.

\section{Basic Fractal Formula}

Let there be a curve (such as coastline), measured by a ruler with length of $\delta$; its length is

$$
L=N \delta \text {, }
$$

where $L$ is the measured length of the curve and $N$ is the times of measurement. However, for coastline such a fractal curve with a self-similarity, $L$ is related to $\delta$; that is,

$$
L=L(\delta)=N \delta=L_{0}\left(\frac{\delta}{L_{0}}\right)^{1-D},
$$

where $L_{0}$ is the length when the curve is integral, a constant; $D$ shows the complexity of the curve, which is fractal dimension, $D \geq 1$. From the above equation, if $D=1$, that is, a smooth curve, $L=L_{0}$; if $\delta=L_{0}$, then $L=L_{0}$; which is consistent with the practical situation.

In (3) and (4), when $\delta \rightarrow 0, L \rightarrow \infty$, therefore, in terms of fractal coastline, measuring the length value is not a good method to characterize coastline, the other appropriate parameters must be sought. In fact, the basic characteristics of fractal are nondifferentiable nature, nonsmoothness, discontinuities, and hierarchy, and the fractal dimension $D$ is able to precisely measure the complexity and the fractal space-filling ability of the object. So, fractal dimension $D$ should represent the essential characteristics of the coastline.

If the fractal object to be examined is not a line, but a point, plate, or body, then formula (3) can be generalized as follows:

$$
A(\delta)=A_{0}\left(\frac{\delta}{\delta_{\max }}\right)^{E-D}
$$

In the formula, when $E=0, A$ and $\delta$ correspond to the number of points (number of lines, such as Cantor set); when $E=1, A$ and $\delta$ correspond to the length; when $E=2, A$, and $\delta$ correspond to the area and length; when $E=3, A$ and $\delta$ correspond to the volume and length; $A_{0}$ means the value of $A$ when the graphics are integer; $\delta_{\max }$ is the size of the maximum length; $D$ is the fractal dimension. Equation (4) is the basic formula of fractal geometry.

Aggregate occupies limit volume in three-dimensional space; therefore, it is three-dimensional, not fractal. However, 

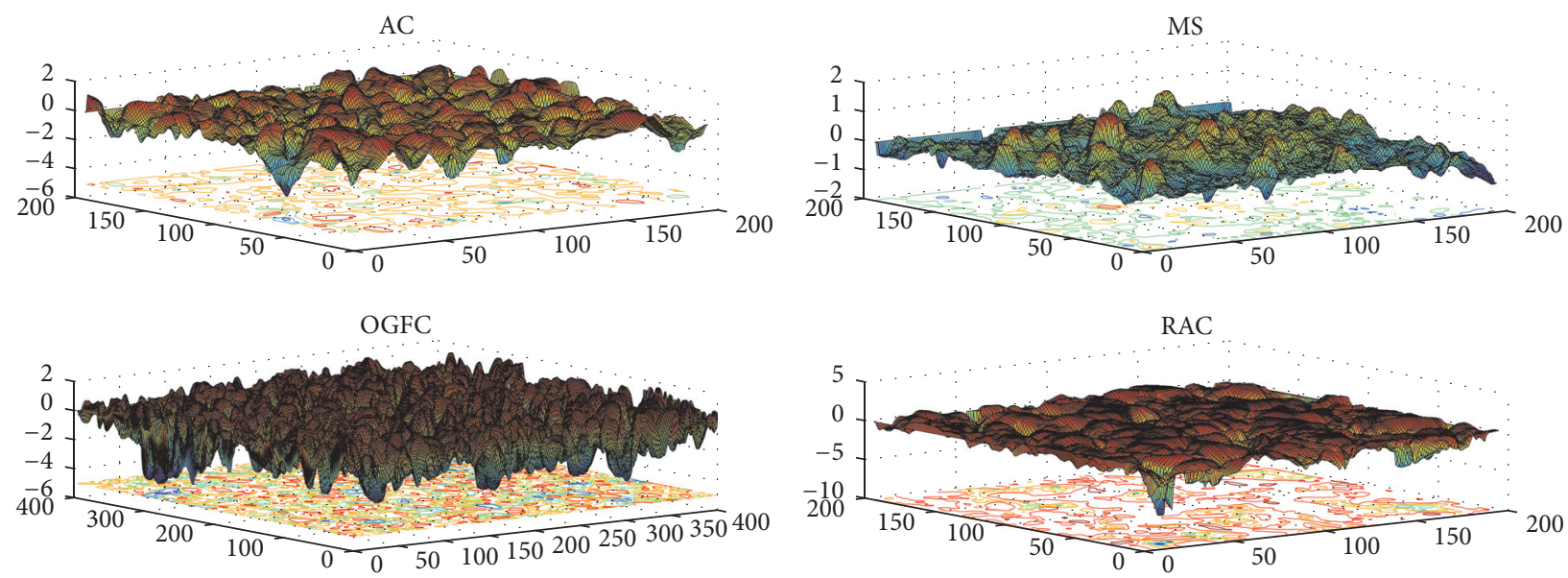

SMA

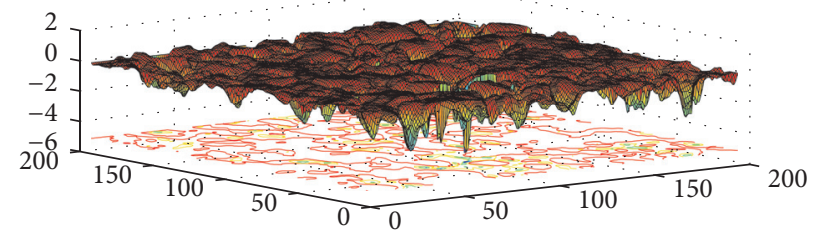

FIGURE 1: Surface textures of five different pavement types.

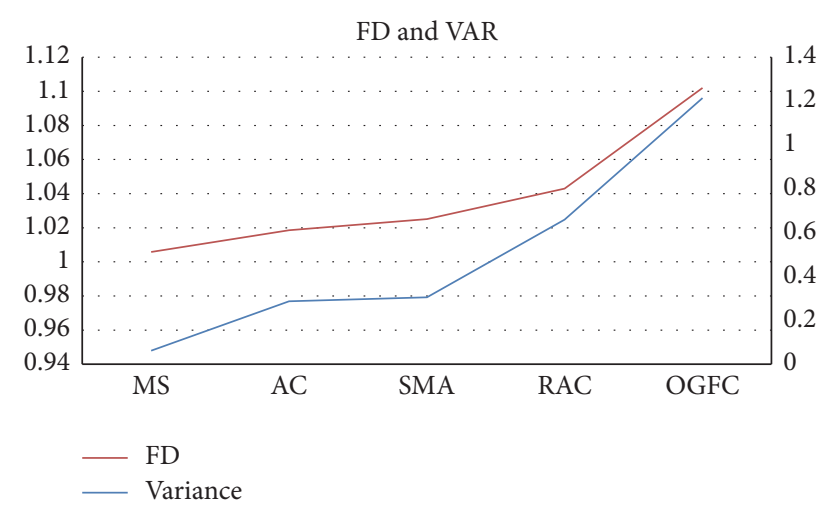

FIGURE 2: The relationship between fractal dimension and variance.

mixed with graded aggregates, aggregates cannot fill the whole occupied three-dimensional space (there is a gap). According to the fractal definition, $t$ 's incapacity to fill the space forms the volume fractal. Hence,

$$
V=V_{0}\left(\frac{\delta}{\delta_{\max }}\right)^{3-D} .
$$

The derivation of (6) is

$$
d V(\delta)=V_{0}\left(\frac{1}{\delta_{\max }}\right)^{3-D} \cdot(3-D) \cdot \delta^{2-D} d \delta,
$$

where $V=$ bulk volume of the aggregate finer than diameter $\delta$, $V_{0}=$ bulk volume of all the aggregate, $\delta=$ aggregate diameter, $\delta_{\max }=$ maximum aggregate diameter, $d V\left(\delta_{\max }\right)=$ the bulk volume of aggregates, respectively, in the interval $(x, x+d x)$, and $D=$ volume fractal dimension of aggregates.
The mass of aggregate with diameter less than $\delta$ is

$$
M(\delta)=\int_{\delta_{\min }}^{\delta} \rho d V(\delta) .
$$

From (5), (6), and (7),

$$
M(\delta)=\frac{\rho V_{0}}{\delta_{\max }^{3-D}}\left(\delta^{3-D}-\delta_{\min }^{3-D}\right) .
$$

Based on the definition of passing rate of aggregates,

$$
P(\delta)=\frac{M(\delta)}{M\left(\delta_{\max }\right)} .
$$

From (8) and (9),

$$
P(\delta)=\frac{\delta^{3-D}-\delta_{\min }^{3-D}}{\delta_{\max }^{3-D}-\delta_{\min }^{3-D}} .
$$

When the minimum particle size of the aggregate $\delta_{\min }$ is very small, which can be ignored, formula (10) can be simplified into

$$
P(\delta)=\left(\frac{\delta}{\delta_{\max }}\right)^{3-D} .
$$

In the previous research, with describing the gradation of the aggregates, the value of $D$ can also be determined. However, it is very difficult to use $D$ to describe gradation, because it is hard to gain the $D$ value, unless the sample is scanned by the $3 \mathrm{D}$ laser scanner, the output data can be used to calculate the fractal dimension. 


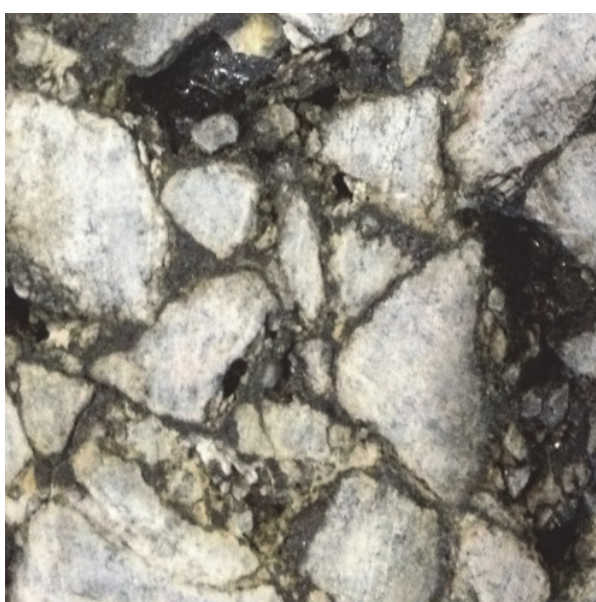

Original

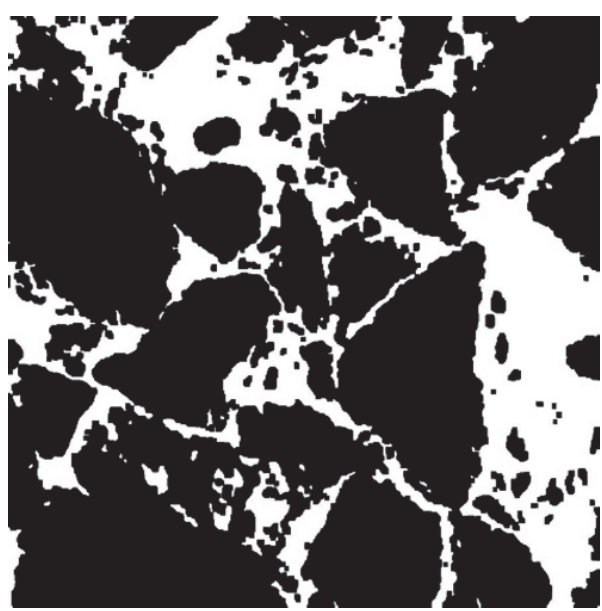

Binary

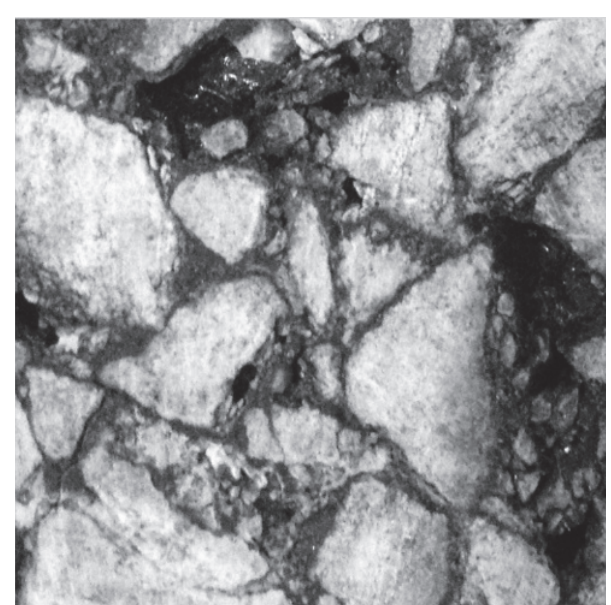

Greyscale

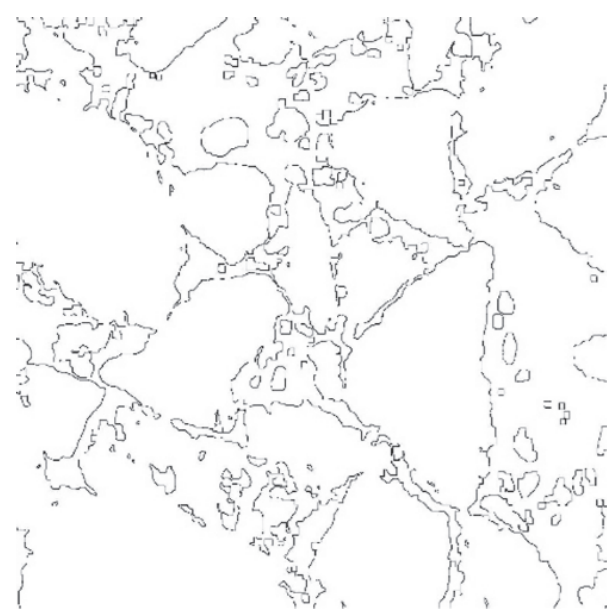

Aggregate contour

FIGURE 3: Image analysis process.

Since the aggregates are not able to fill the whole area space, it also possesses fractal characteristics. And the derivation of volume can be area

$$
d V=A=A_{0}\left(\frac{\delta}{\delta_{\max }}\right)^{2-D_{a}} .
$$

$A=$ area of the aggregates size less than $\delta$ on the surface and $D_{a}=$ area fractal dimension of $2 \mathrm{D}$ image.

According to the definition of volume,

$$
d V_{0}=\frac{M_{0}}{\rho} d P,
$$

where $M_{0}=$ the total mass of the aggregate, $\rho$ = the density of aggregates, and $P=$ the passing rate.

In the interval $(\delta, \delta+\Delta)$, the fractal volume is

$$
d V=\left(\frac{\delta}{\delta_{\max }}\right)^{3-D} d V_{0} .
$$

Based on (12) and (14),

$$
\left(\frac{\delta}{\delta_{\max }}\right)^{3-D} \frac{M_{0}}{\rho} d P=A_{0}\left(\frac{\delta}{\delta_{\max }}\right)^{2-D_{a}} .
$$

TABLE 2: Gradation of the sample.

\begin{tabular}{lcccccc}
\hline Sieve size & $1.5^{\prime \prime}$ & $1^{\prime \prime}$ & $0.5^{\prime \prime}$ & $\# 4$ & $\# 8$ & $\# 200$ \\
\hline Passing rate & 100 & 95 & 47 & 3 & 2 & 1.4 \\
\hline
\end{tabular}

From the definition of passing rate,

$$
P(\delta)=\frac{M(\delta)}{M_{0}}=\left(\frac{\delta}{\delta_{\max }}\right)^{D-D_{a}} .
$$

From (5), we have

$$
P(\delta)=\left(\frac{\delta}{\delta_{\max }}\right)^{(1 / 2)\left(3-D_{a}\right)} .
$$

Through the above equations, the relationship between passing rate and area fractal dimension can be built up.

\section{The Verification of the Equation}

5.1. Gap Graded Aggregate. Table 2 shows the gap gradation information of one laboratory sample. 


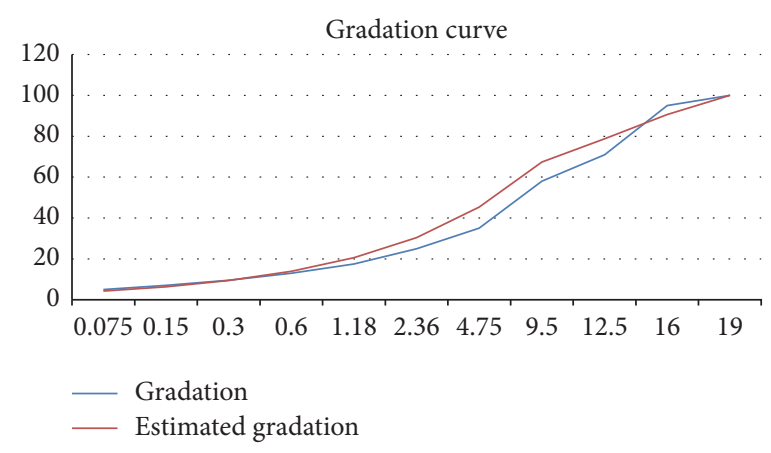

FIGURE 4: Gradation curve.
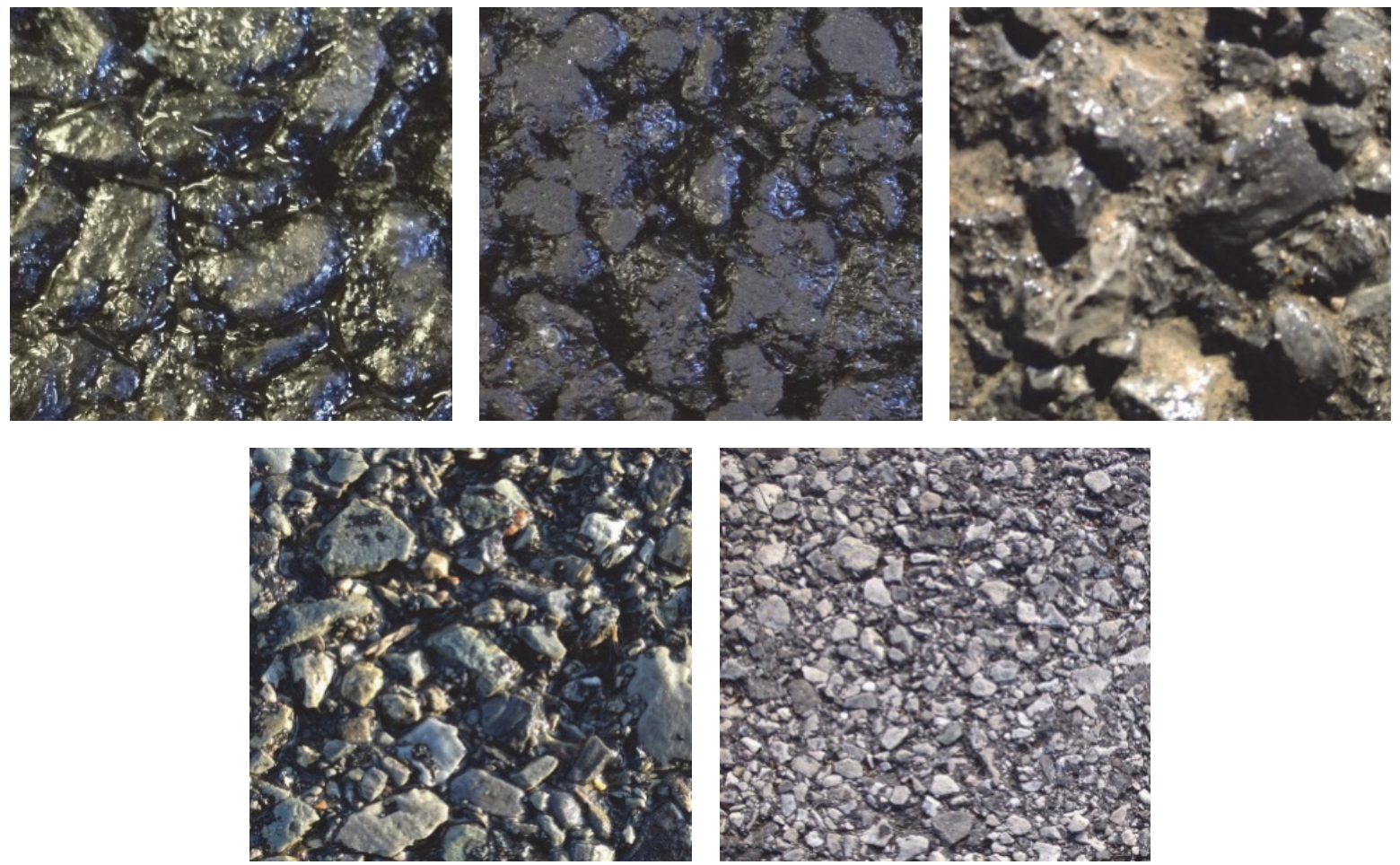

FIGURE 5: Surface texture image of 5 in situ samples.

In the light of (11), the logarithm on both sides of equation is shown in

$$
\log P(\delta)=\frac{1}{2}\left(3-D_{a}\right) \log \left(\frac{\delta}{\delta_{\max }}\right) .
$$

$(1 / 2)\left(3-D_{a}\right)=$ slope of the plot of $\log P(\delta)-g\left(\delta / \delta_{\max }\right)$ curve. Through the aforementioned calculation, $D_{a}=1.51$.

According to the area fractal dimension calculation in MATLAB program, $D_{a}=1.73$ which is a little different from the one obtained by gradation curve. It can be seen that to describe gradation via $D_{a}$ in equation $P(\delta)=$ $\left(\delta / \delta_{\max }\right)^{(1 / 2)\left(3-D_{a}\right)}$ is not feasible in this case, because this sample is in gap gradation and some of the sieve sizes are absent.

5.2. Continuous Graded Aggregate. Table 3 is the gradation of the CT scan sample from Zhang's [16] paper.
The area fractal dimension of the continuous gradation sample is 1.86 via the calculation of the MATLAB program. Meanwhile, the $D_{a}$ value obtained from (13) is 1.85 which is quite similar to the prior one of 1.86 . Figure 4 is the comparison of gradation curve between the original data and the gradation from the equation.

As a result, (13) is only relevant to the continuous graded aggregates, not for gap gradation. Table 4 shows the BPN value and contour fracture dimension of aggregate. As seen, the $2 \mathrm{D}$ contour fractal dimension is able to represent the contour complexity and roughness of the aggregates. Therefore, the $2 \mathrm{D}$ contour fractal dimension can also characterize the friction and roughness of pavement texture due to the random placement of the aggregates in paving process.

In this report, British Pendulum friction instrument is used to test BPN value of five samples, as shown in Figure 5. The $2 \mathrm{D}$ image contour fractal dimensions are also calculated. 
TABLE 3: Gradation of the CT sample.

\begin{tabular}{lcccccccccccc}
\hline Sieve size & $3 / 4^{\prime \prime}$ & $5 / 8^{\prime \prime}$ & $0.5^{\prime \prime}$ & $3 / 8^{\prime \prime}$ & $\# 4$ & $\cdots$ & $\# 8$ & $\# 16$ & $\# 30$ & $\# 50$ & $\# 100$ & $\# 200$ \\
\hline Passing rate (\%) & 100 & 95 & 71 & 58 & 35 & $\cdots$ & 25 & 17.5 & 13 & 9.5 & 7 & 5 \\
\hline
\end{tabular}

TABLE 4: BPN value and contour fractal dimension of aggregate.

\begin{tabular}{lccccc}
\hline & Sample 1 & Sample 2 & Sample 3 & Sample 4 & Sample 5 \\
\hline BPN & 74 & 80 & 100 & 102 & 105 \\
Contour fractal dimension & 1.4287 & 1.44 & 1.515 & 1.555 & 1.5634 \\
\hline
\end{tabular}

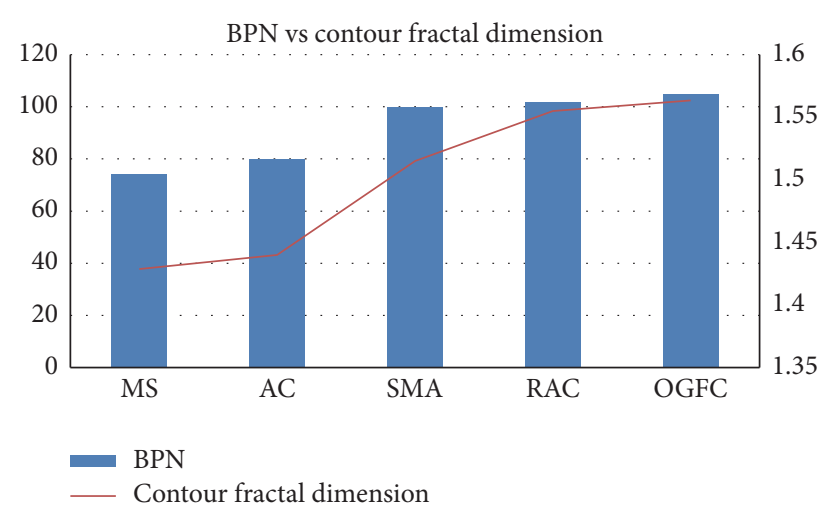

FIGURE 6: Relationship between BPN and contour fractal dimension.

From Figure 6, it turns out that the contour fractal dimension increases with the BPN value. To some degree, the contour fractal dimension can be used as a parameter to characterize the friction and roughness of pavement surface. Hence, when describing the 2D image of the surface texture of aggregates, the BPN or friction coefficients are able to be obtained. It is a simple way to quantify the pavement surface roughness.

The nonlinear regression between BPN and the contour fractal dimension was built in least squares method in the light of the regression result:

$$
\mathrm{BPN}=-1579 \times \mathrm{FD}^{2}+4941 \times \mathrm{FD}-3761 .
$$

The $R$-square is 0.9937 which means the BPN and FD correlate very well in the nonlinear regression.

\section{Conclusions}

Pavement surface texture has roughly repetitive features and self-similarity, representing the fractal characterizes. Fractal geometry provides a relevant method to describe the irregularities and complexity of pavement surface texture, being widely used in pavement engineering. In this paper, the quantitative relationship between area fractal dimension and gradation is built, and the calculation formula of area fractal dimension of aggregate distribution characteristics can be deduced via the presentation of their mass fractal characteristic function. In addition, there is a good nonlinear relation between the BPN and contour fractal dimension.

\section{Competing Interests}

The authors declare that they have no competing interests.

\section{Acknowledgments}

The research performed in this paper is supported by the National Natural Science Foundation of China (no. 51308042) and the Fundamental Research Funds for the Central Universities (06500036).

\section{References}

[1] B. B. Mandelbrot, Fractals and Chaos, Springer, Berlin, Germany, 2004.

[2] B. B. Mandelbrot, The Fractal Geometry of Nature, Macmillan, New York, NY, USA, 1983.

[3] J.-F. Gouyet, Physics and Fractal Structures, Masson, Paris, France, Springer, New York, NY, USA, 1996.

[4] J. R. Price and M. H. Hayes, "Resampling and reconstruction with fractal interpolation functions," IEEE Signal Processing Letters, vol. 5, no. 9, pp. 228-230, 1998.

[5] S. Arasan, E. Yener, F. Hattatoglu, S. Akbulut, and S. Hinislioglu, "The relationship between the fractal dimension and mechanical properties of asphalt concrete," International Journal of Civil and Structural Engineering, vol. 1, no. 2, pp. 165-170, 2010.

[6] M. M. Villani, A. Scarpas, A. de Bondt, R. Khedoe, and I. Artamendi, "Application of fractal analysis for measuring the effects of rubber polishing on the friction of asphalt concrete mixtures," Wear, vol. 320, no. 1, pp. 179-188, 2014.

[7] W. Dong, X. Guo, and L. Gu, "Study on the fractal gradation optimization of ATB-25," in Proceedings of the 2nd International Conference on Electronic \& Mechanical Engineering and Information Technology (EMEIT '12), Shenyang, China, September 2012.

[8] H. Liu and L. Wang, "Fractal dimension analysis of the fine aggregate gradation of interlocking skeleton asphalt mixture," Journal of Wuhan University of Technology-Materials Science Edition, vol. 26, no. 3, pp. 567-572, 2011.

[9] Y. Hou, L. Wang, P. Yue, T. Pauli, and W. Sun, "Modeling mode I cracking failure in asphalt binder by using nonconserved phasefield model," Journal of Materials in Civil Engineering, vol. 26, no. 4, pp. 684-691, 2014.

[10] Y. Hou, F. Sun, W. Sun, M. Guo, C. Xing, and J. Wu, "Quasibrittle fracture modeling of preflawed bitumen using a diffuse interface model," Advances in Materials Science and Engineering, vol. 2016, Article ID 8751646, 7 pages, 2016.

[11] Y. Hou, W. Sun, P. Das et al., "Coupled navier-stokes phase-field model to evaluate the microscopic phase separation in asphalt 
binder under thermal loading," Journal of Materials in Civil Engineering, vol. 28, no. 10, Article ID 04016100, 2016.

[12] Y. Hou, P. Yue, L. Wang, and W. Sun, "Fracture failure in crack interaction of asphalt binder by using a phase field approach," Materials and Structures, vol. 48, no. 9, pp. 2997-3008, 2015.

[13] Y. Hou, L. Wang, T. Pauli, and W. Sun, "Investigation of the asphalt self-healing mechanism using a phase-field model," Journal of Materials in Civil Engineering, vol. 27, no. 3, Article ID 04014118, 2015.

[14] O. M. Bruno, R. de Oliveira Plotze, M. Falvo, and M. de Castro, "Fractal dimension applied to plant identification," Information Sciences, vol. 178, no. 12, pp. 2722-2733, 2008.

[15] R. F. Voss, "Fractals in nature: from characterization to simulation," in The Science of Fractal Images, pp. 21-70, Springer, New York, NY, USA, 1988.

[16] J. Zhang, Research on characterization of air void distribution and its fine description in porous drainage asphalt mixture [M.S. thesis], Chang'an University, 2008. 

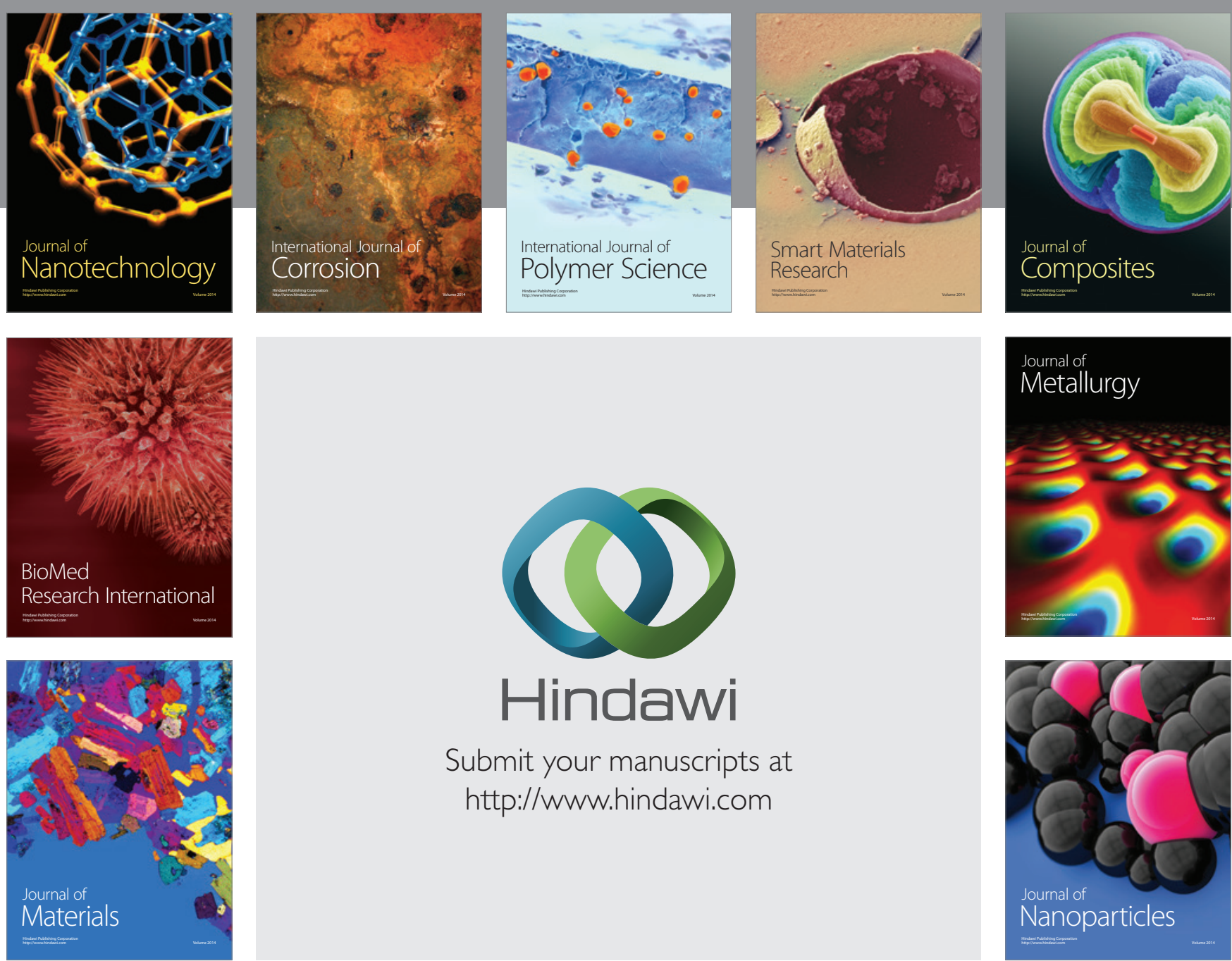

\section{Hindawi}

Submit your manuscripts at

http://www.hindawi.com

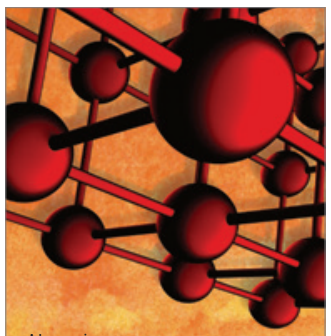

Materials Science and Engineering
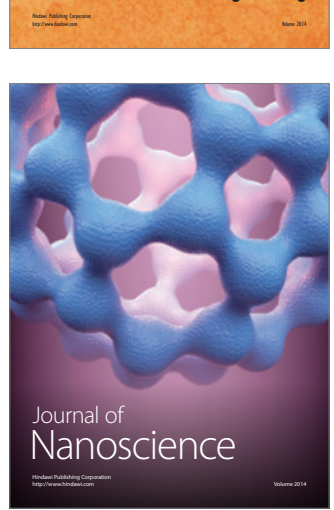
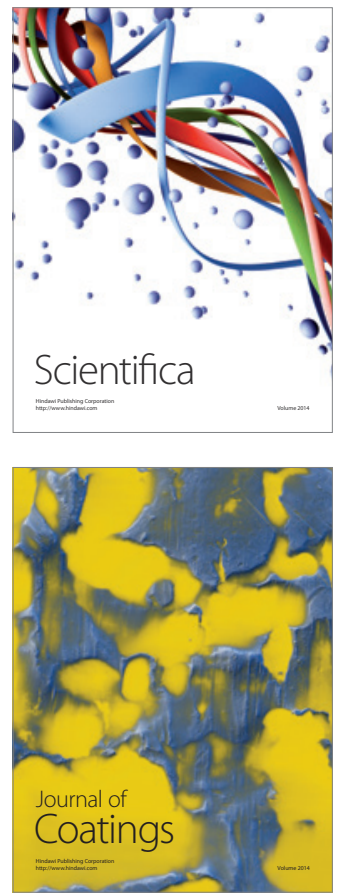
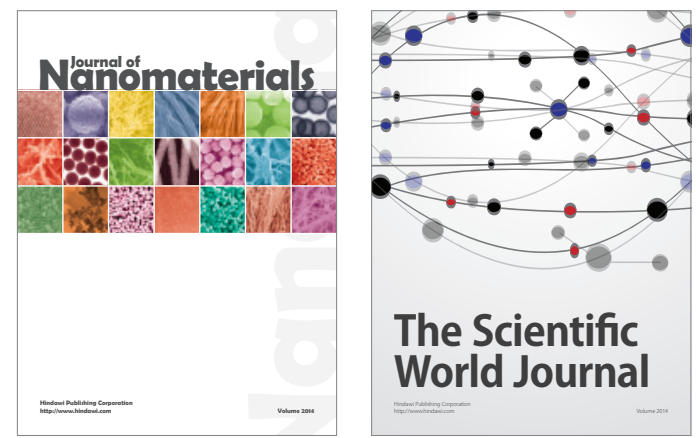

The Scientific World Journal
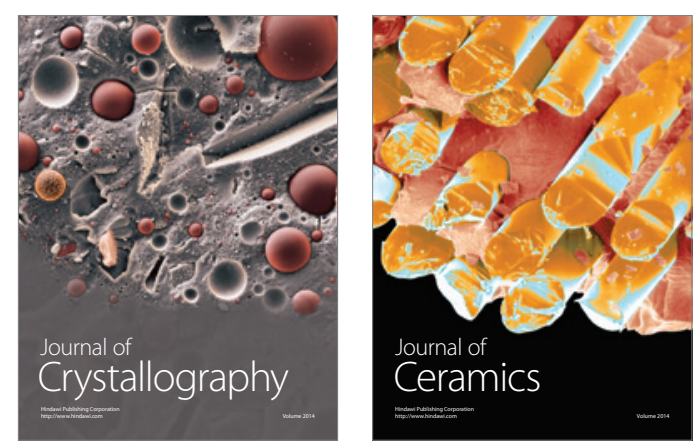
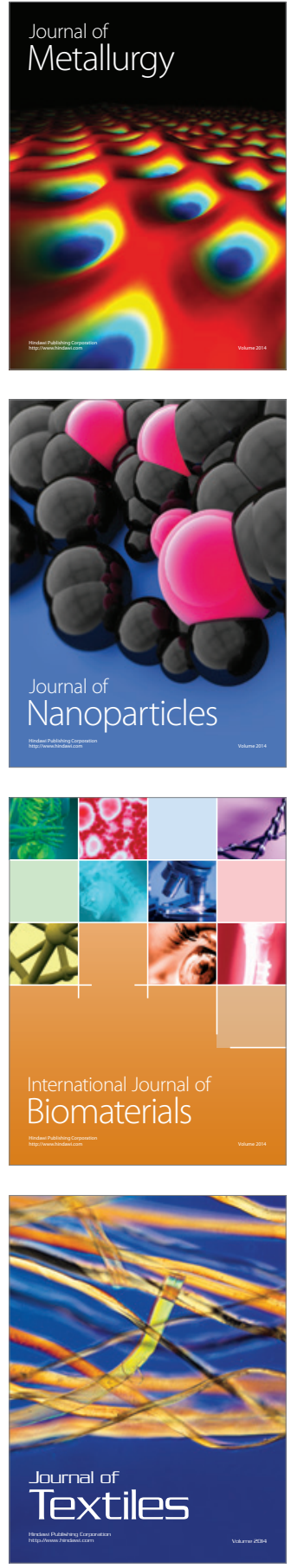